\title{
Recent Progress in Fiber Optofluidic Lasing and Sensing
}

\author{
Xi YANG ${ }^{1}$, Chaoyang GONG ${ }^{1,2}$, Yiling LIU ${ }^{1}$, Yunjiang RAO ${ }^{1,3}$, \\ Mateusz SMIETANA ${ }^{4}$, and Yuan $\mathrm{GONG}^{1^{*}}$ \\ ${ }^{1}$ Key Laboratory of Optical Fiber Sensing and Communications (Ministry of Education of China), University of \\ Electronic Science and Technology of China, Chengdu 611731, China \\ ${ }^{2}$ School of Electrical and Electronic Engineering, Nanyang Technological University, 50 Nanyang Avenue, 639798, \\ Singapore \\ ${ }^{3}$ Research Center for Optical Fiber Sensing, Zhejiang Laboratory, Hangzhou 310000, China \\ ${ }^{4}$ Warsaw University of Technology, Institute of Microelectronics and Optoelectronics, Koszykowa 75, 00-662 Warsaw, \\ Poland \\ *Corresponding author: Yuan GONG $\quad$ E-mail: ygong@uestc.edu.cn
}

\begin{abstract}
Fiber optofluidic laser (FOFL) integrates optical fiber microcavity and microfluidic channel and provides many unique advantages for sensing applications. FOFLs not only inherit the advantages of lasers such as high sensitivity, high signal-to-noise ratio, and narrow linewidth, but also hold the unique features of optical fiber, including ease of integration, high repeatability, and low cost. With the development of new fiber structures and fabrication technologies, FOFLs become an important branch of optical fiber sensors, especially for application in biochemical detection. In this paper, the recent progress on FOFL is reviewed. We focuse mainly on the optical fiber resonators, gain medium, and the emerging sensing applications. The prospects for FOFL are also discussed. We believe that the FOFL sensor provides a promising technology for biomedical analysis and environmental monitoring.
\end{abstract}

Keywords: Optical fiber sensors; optofluidic laser; microstructured optical fiber; optical microcavity; biochemical sensors

Citation: Xi YANG, Chaoyang GONG, Yiling LIU, Yunjiang RAO, Mateusz SMIETANA, and Yuan GONG, "Recent Progress in Fiber Optofluidic Lasing and Sensing," Photonic Sensors, 2021, 11(2): 262-278.

\section{Introduction}

Optical fiber sensors have been widely applied in a variety of fields, owing to their immunity to electromagnetic interference, capability for operation in harsh environments and at ultra-long distance without power supply, high robustness, high repeatability, and small cross-sectional size [1-3]. Fiber optic gyroscope can be treated as a very successful example of an optical fiber sensor [4].
Also fiber Bragg grating (FBG) sensors have been successfully commercialized for strain, temperature, and pressure monitoring [5-7]. Distributed optical fiber sensors have been breaking the ground in many fields including oil/gas exploration, earthquake monitoring, safety inspection, and structural health monitoring [8-11]. Most successes of optical fiber sensors are for physical parameter sensing and comparatively, optical fiber biochemical sensing is still on the way.

Received: 18 February 2021 / Revised: 9 March 2021

(C) The Author(s) 2021. This article is published with open access at Springerlink.com

DOI: $10.1007 / \mathrm{s} 13320-021-0622-9$

Article type: Review 
Thanks to the high sensitivity, small size, and biocompatibility, fiber biochemical sensors have been explored to monitor biochemical information such as the refractive index (RI), concentration, molecular structure, molecular weight, and interaction between molecules [12-14]. With the development of advanced fabrication technologies, different types of fiber gratings, including tilted FBG and long-period fiber gratings, have been presented for biochemical sensing [15]. The fiber-optic micro-interferometers such as Mach-Zehnder (MZ) and Fabry-Perot (FP) interferometers can achieve very high sensitivity based on phase detection [16]. One of the most popular sensing principles is to employ the evanescent wave of the optical fiber to interact with the surrounding analytes. A number of optical micro-/nano-fibers or tapered optical fibers have been designed to enhance these interactions [17]. Also surface plasmonic resonance (SPR) based on optical fibers have been extensively investigated for biochemical analysis and have shown ultrahigh sensitivity [18]. The sensors make it possible to monitor the kinetics of biomolecule binding in real time. Benefitted from the rich luminescent materials, fluorescence based fiber biochemical sensors can detect biochemical information through the principles of fluorescent resonance energy transfer (FRET), or the detection of intensity, polarization, or lifetime [19].

Laser-based biochemical sensors can offer a great enhancement of light-analyte interaction due to the amplification of optical microcavity and laser generation process, enabling a high signal-to-noise ratio and high sensitivity [20-22]. In this paper, we review the recent progress in fiber optofluidic lasers (FOFLs) and their applications for biochemical sensing. A brief overview of the FOFL technological components, especially the microresonator structure and gain materials are provided. Typical applications using FOFLs for biochemical sensing, cell labeling, and tissue mapping are also given. Finally, we discuss the future directions of the FOFL development.

\section{Optical feedback in FOFLs}

\subsection{Fabry-Perot cavity}

Fabry-Perot cavities are the most frequently used optical feedback structures for traditional lasers, due to their numerous advantages of simple structure and ease of fabrication. For example, metal or dielectric films, or even two surfaces with Fresnel reflections at optical fiber end-face can be used for the formation of the FP cavity. Figure 1(a) shows the fiber FP cavity proposed by Zhou et al. [23]. It was achieved by coating a gold film on the end-faces of single-mode fibers using a physical vapor deposition method. Two capillaries served as microfluidic channels and were used to ensure the alignment of the end-faces. The lasing wavelength can be tuned from $564 \mathrm{~nm}$ to $581 \mathrm{~nm}$ by changing the cavity length from $3 \mathrm{~mm}$ to $20 \mathrm{~mm}$. Employing the easy mixture property of liquid, Kou et al. [24] demonstrated a dual-color FOFL by mixing two kinds of dyes as gain medium. Fast switching of FOFL was also achieved by the alternation of different dye droplets in oil flow and exhibited a single-longitudinal-mode emission [Fig. 1(b)] [25].

The methods for constructing a fiber FP cavity are similar to those based on reflective mirrors. However, the small cross-section of fiber makes a weak adhesion of reflective films, even if an intermediate adhesion-improving film like titanium is deposited. Hence, an all-fiber optofluidic laser was developed by employing the Fresnel reflections as optical feedback [26]. Even with only 4\% reflection, lasing emission was observed by using Rhodamine 640 (Rh640) as the gain medium and the threshold can be less than $1 \mu \mathrm{J}$ per single pulse. Eliminating the coating process makes it simpler and cost-effective, however, the additional plugs for withdrawing the liquid results in a complex structure in the microfluidics part. 


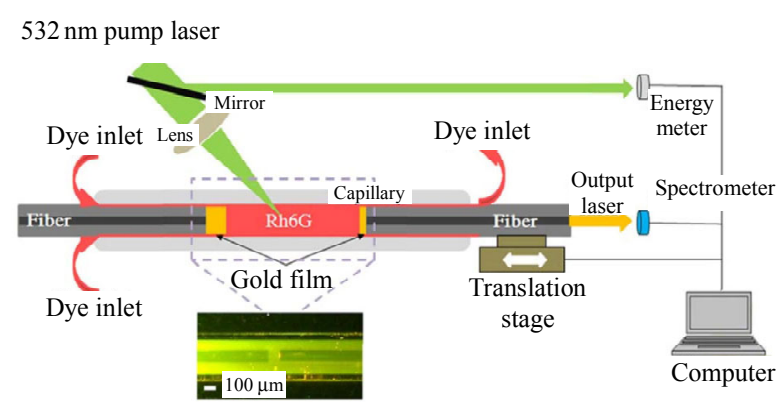

(a)

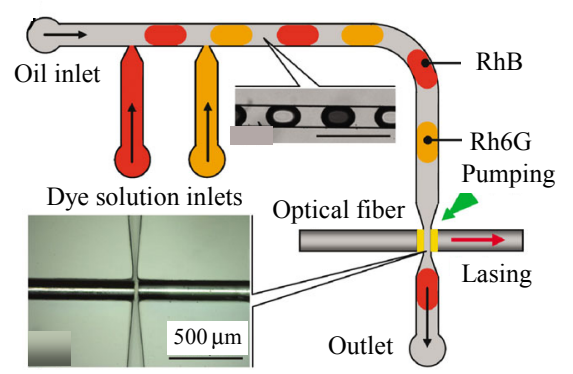

(b)

Fig. 1 Schematic diagrams of fiber optofluidic lasers based on fiber FP cavities: (a) FOFL with the tunable wavelength [23] and (b) fast switching of FOFL via the alternation of droplets that contain two different dyes [25].

Fiber FP cavity and optical fiber itself have the spectral filtering effect, enabling a single-mode laser emission. By using a single-mode fiber, instead of the multimode fiber, to deliver the pump laser, the number of transverse modes can be greatly reduced [26]. Through shortening the fiber FP cavity length down to $18 \mu \mathrm{m}$, a single-mode laser with a full width at half maximum (FWHM) of $120 \mathrm{pm}$ was demonstrated [25]. The FWHM of a single-mode FOFL was further reduced to $53 \mathrm{pm}$ by using the filtering effect of the cross-section of a twin-hole microstructured optical fiber (MOF) [27].

\subsection{Fiber microring resonator}

On-chip microring resonators have been extensively investigated and used for ultrahigh sensitive detection of a single particle or molecule, and for the development of compact optical frequency combs [28-30]. Different from the on-chip resonator on a planar substrate, fiber micro-resonators are based on the microring in the cross-section of optical fibers. There are many unique properties of the fiber microring resonators, including high reproducibility, ease of fabrication, low cost, and natural integration of microfluidic channel. The most promising one is the highly reproducible microrings achieved by the precise control of geometry during the fiber drawing process.

\subsubsection{Telecom optical fiber}

With the fast development of optical fiber communications, telecom optical fibers have been mass-produced by commercial fiber draw towers at a very low cost. During the fiber drawing process, the surface tension makes the optical fiber form a round geometry and a smooth surface. The round outer surface can serve as a microring resonator to provide optical feedback for lasing and its $Q$-factor can be as high as $10^{7}[31]$. The $Q$-factor relies on the properties of the outer surface, including smoothness, roundness, and absorption, but regardless of the axial waveguide properties of the fiber core, including single- or multimode. There are two main structures of FOFLs based on telecom optical fibers. One is by using a gain medium in the bulk solution and the other is based on a gain layer attached to the outer surface of the fiber.

The fiber-in-capillary structure was often used for demonstrating the FOFLs with bulk liquid gain [32-34]. In this structure, the fiber microring resonator provides optical feedback and the glass capillary is used as a microfluidic channel. The capillary is also helpful to limit the lateral volume of the bulk gain and consequently to reduce the fluorescent background.

By using the surface conjugation, Chen et al. [35] have demonstrated a fiber optofluidic laser with a single layer of gain molecules (Fig. 2). All the gain molecules can interact with the evanescent wave, strongly enhancing the light-matter interaction and further improving the laser efficiency and threshold. 
Compared with bulk gain medium, this kind of FOFL greatly reduces the number of gain molecules and thus the fluorescent background. It is promising for surface-based bioassays. Digital deoxyribonucleic acid (DNA) detection was performed by using this technology [36].

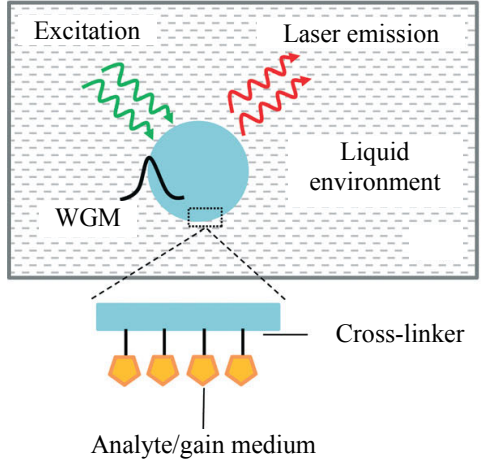

(a)

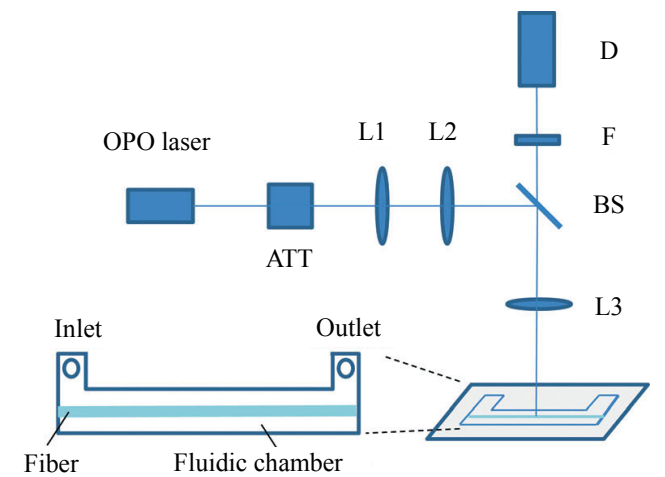

(b)

Fig. 2 Fiber optofluidic laser based on telecom optical fiber with a single layer of gain molecules [35]: (a) cross-sectional view of the FOFL in liquid and (b) schematic of the experimental setup. ATT: attenuator; L1/L2/L3: lenses; BS: beam splitter; F: filter; D: detector.

\subsubsection{Hollow optical fiber}

Hollow optical fiber (HOF) can serve as a liquid-core optical ring resonator (LCORR) for lasing when being filled with liquid gain [Fig.3(a)]. It also acts as a perfect one-dimensional microfluidic channel withdrawing the liquid into the HOF by the capillary action.

For the thin-walled HOF, light circulates in the thin wall of the HOF and interacts with the liquid gain via evanescent wave [Fig.3(b)] [37]. The laser emission can be collected by either tapered fiber or free-space optics. By using an array of tapered fiber, it is available to collect laser emission from different sections of the HOF. The $Q$-factor can reach $\sim 10^{7}$ which leads to a low threshold [38].

Thick-walled HOF can also be employed for optofluidic lasers, when the RI of the gain solution exceeds that of the silica [39]. Total internal reflections in the inner wall provide optical feedback. Owing to the strong overlap between the resonant mode and the gain medium, a low threshold is achievable by using $\mathrm{R} 6 \mathrm{G}$ in quinolone as gain medium [40].

\subsubsection{Microstructured optical fiber}

Microstructured optical fiber has air holes in its cross-section, which can serve as microfluidic channel for liquid gain. The microstructures provide

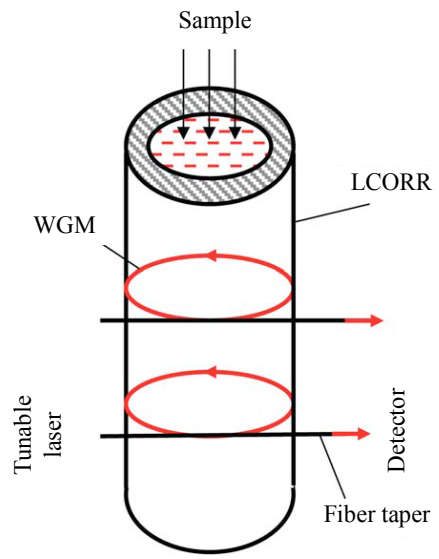

(a)

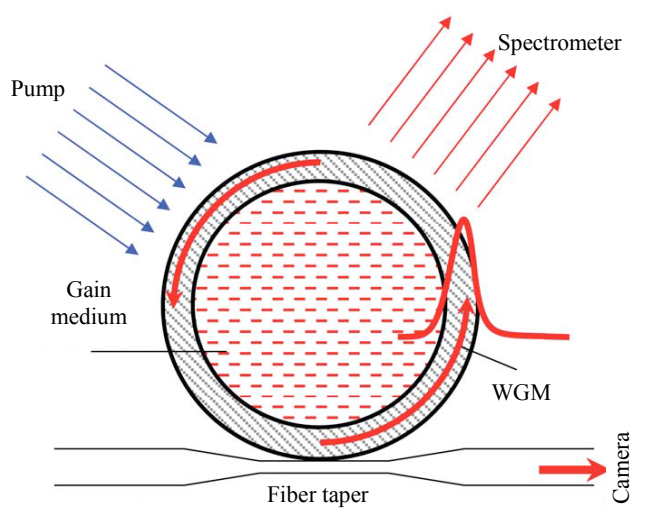

(b)

Fig. 3 FOFL based on thin-walled hollow optical fiber [37]: (a) structure of LCORR and (b) experimental setup for FOFL with fiber taper to evanescently couple out the laser emission. 
a large surface-to-volume ratio for immobilizing biomolecules to achieve high sensitivity. The resonance mechanism of MOF-based microcavity depends on its structure [41-43]. For example, Li et al. [44] demonstrated a fiber optofluidic laser based on hollow-core MOF [HC-MOF, Fig.4(a)]. A 370-nm-thick silica microring supported whispering-gallery-mode (WGM) to provide optical feedback for lasing [Fig.4(b)]. A low threshold of $185 \mathrm{~nJ} /$ pulse was demonstrated by using $3 \mathrm{mM}$ R6G in ethanol as the gain medium. The microring resonator embedded in the MOF made the laser output immune to the perturbation from the ambient environment, including changes of humidity, or contamination on the outer surface.

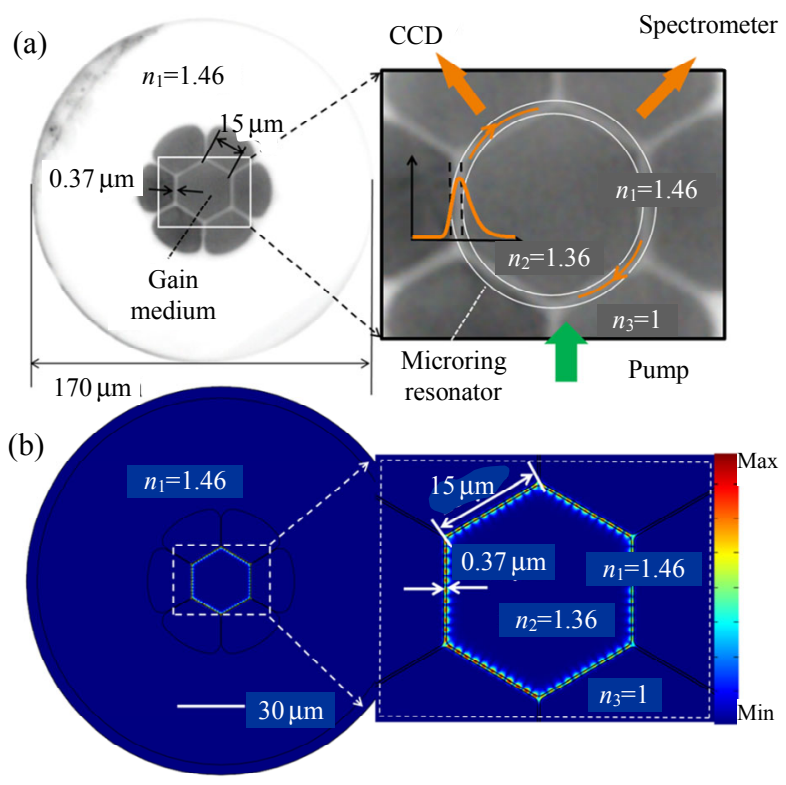

Fig. 4 FOFL based on a MOF [44]: (a) optical micrograph of the cross-section of the HC-MOF and (b) simulated light field distribution of high order mode supported by the dye-filled HC-MOF.

\section{Gain materials}

Activated gain materials can provide gain for optofluidic lasing via stimulated emission. Various kinds of luminescent materials, including organic dyes, rare-earth dopants, quantum dots (QDs), polymer dots, upconversion materials, biomaterials, and semiconductors, have been demonstrated as gain medium for lasing at a different wavelength ranging from ultra-violet (UV) to infrared [45-51]. Most gain materials can combine with the optical fiber microresonators discussed in Section 2, and their emission properties can be tuned by using different solvents. Here, we introduce organic dyes, nanocrystals (NCs), and biological gain materials for fiber optofluidic lasing.

\subsection{Organic dyes}

Organic dyes have been the most commonly used gain materials for liquid lasers, due to their high quantum yield, high brightness, broad spectrum coverage, low threshold, and low cost. However, the photobleaching of organic dye is harmful to the achievement of stable laser output. This adverse effect could be reduced by using a continuous flow of dye solution, or decreasing the repetition rate of the pump laser, even a single-pulse pump.

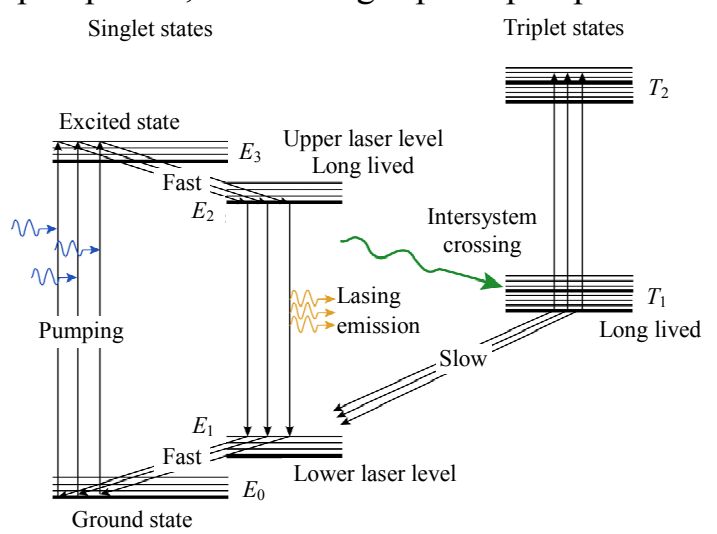

(a)

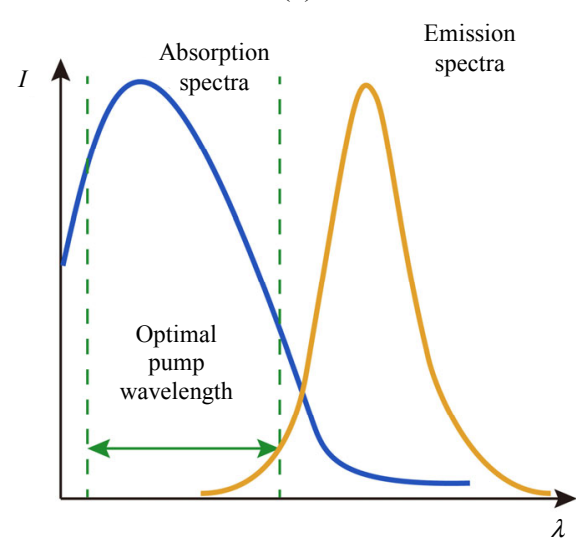

(b)

Fig. 5 Typical energy levels of (a) a dye molecule and (b) corresponding absorption and emission band. 
Generally, laser dyes are complex organic compounds containing long chains of conjugated double bonds, which lead to many vibrational and rotational levels within a single electronic state [52, 53]. Hence, organic dyes have strong and wide absorption bands in the UV and visible regions. As shown in Fig. 5(a), upon optical excitation to upper states $\left(E_{3}\right)$, the dye molecules rapidly relax to the bottom of the first-excited single state $\left(E_{2}\right)$ within picoseconds via non-radiative vibrational relaxation and then relax back to the ground state $\left(E_{1}\right)$ by emitting a photon via fluorescence on a timescale of nanoseconds. When the number of dye molecules in $E_{2}$ is larger than $E_{1}$ (population inversion), the stimulated emission occurs, thus providing optical amplification for lasing. The details of the lasing process using a laser dye were described in [52].

Thanks to the reconfigurability of microfluidics, dye lasers cover a spectral range of hundreds of nanometers, making it promising as an on-chip laser source as well as wavelength multiplexing. The wavelength of dye lasers can be tuned in several ways. One is to change the organic dyes directly [54]. Laser dyes are commercially available with emission wavelength ranging from $\mathrm{UV}$ to near-infrared (NIR), which are provided by Exciton Inc., Radiant Dye Laser \& Accessories GmbH, etc. The laser wavelength can be precisely tuned by adjusting the concentration of the organic dye or using different types of solvents. For example, using R6G as a gain medium, the laser emission was tuned from $560 \mathrm{~nm}$ to $610 \mathrm{~nm}$ by changing the solvents of ethanol, tetraethylene glycol, methanol, and quinolone [31, 38, 55, 56]. Moon et al. [31] demonstrated a tunable band of $35 \mathrm{~nm}$ by altering the concentration of $\mathrm{R} 6 \mathrm{G}$ in a mixed solvent of ethanol and ethylene glycol. In addition, the laser wavelength can be modulated by the optical property of organic dye, e.g., the reabsorption effect. By adjusting the cavity length of the fiber FP cavity from $3 \mathrm{~mm}$ to $20 \mathrm{~mm}$, the laser wavelength can be shifted by $18 \mathrm{~nm}$ [23]. Similarly, the RI of dye solution would influence the resonance properties of the optical fiber microcavities and thus induce the spectral shift of lasing [57, 58]. Combining with FRET, the tunability of laser wavelength can be further enlarged. Gong et al. [59] achieved a $250 \mathrm{~nm}$ wavelength tunability based on microfluidics and FRET.

Lasing can also be achieved based on dyes solidified in a film. A thin film with a thickness of $1 \mu \mathrm{m}-2 \mu \mathrm{m}$ concentrates the dye molecules in a small volume, which improves the pumping efficiency and reduces the fluorescence background. In sensing applications, the solid gain medium could be spatially separated with the biosample to minimize the undesired effects on biosamples. They were demonstrated by coating a thin film of the dye-doped polymer or the conducting polymer itself either on the inner surface of HOF or on the outer surface of telecom optical fiber [60-64]. Polymers including polystyrene, polyvinyl-pyrrolidone, and poly (benzyl methacrylate) are attractive frame materials for hosting dyes [61, 65-67].

\subsection{Nanocrystals}

Nanocrystals, a type of nanomaterials with at least one-dimension smaller than $100 \mathrm{~nm}$, exhibit strongly size-dependent optical and electrical properties [68]. It provides gain for lasing with the principle of quantum traps. For the QDs, an electron-hole pair generates after the absorption of energy, and a photon is produced when the electron-hole pair recombines. Compared to organic dyes, NCs serve as a promising gain medium thanks to their high photostability and large Stokes shift [69, 70]. NCs often have a large absorption cross-section, which is critical for low threshold lasing. The pump wavelength is flexible for NCs as long as the photon energy is higher than its band gap, while for dyes it must fall in the narrow absorption band. Their emission wavelengths can be tuned by the crystal size or their composition [71]. Usually, the emission spectra of NCs are narrow, so that NCs are capable 
of multiplexing applications such as multi-color labeling or cell tagging [72, 73].

Lasing action of NCs has been extensively investigated in solid-state laser, however, its research for lasing in a liquid is scarce. Several factors may influence its lasing efficiency in liquid, including the non-radiative Auger recombination, the loading fraction of NCs in solution, and the photo-induced absorption associated with the surface or interface defects [74]. Nevertheless, several NC-based FOFLs have already been demonstrated, such as CdSe/ZnS QDs in glycerol/ water solution, $\mathrm{CdZnS} / \mathrm{ZnS}$ QDs in toluene, and carbon dots in PEG $[48,75,76]$. The first FOFL using $\mathrm{NCs}$ was implemented by infiltrating $\mathrm{CdSe} / \mathrm{ZnS}$ QRs hexanes solution into a fiber-in-capillary microcavity, in which the high $Q$-factor of the telecom optical fiber provides the necessary conditions to achieve lasing and overcome the competing non-radiative loss [77]. Due to the high photostability of NCs, Wei's group [78] developed an azimuthally polarized radial laser emission by filling $\mathrm{CdSe} / \mathrm{CdZnS} / \mathrm{ZnS}$ QDs hexane solution in photonic bandgap fiber, offering a solution for omnidirectional display and phototherapy with minimal invasion. Lasing with aqueous QDs is crucial for their applications in biomedical environments. Kiraz et al. [79] have demonstrated low-threshold $\left(0.1 \mu \mathrm{J} / \mathrm{mm}^{2}\right)$ lasing emission from aqueous $\mathrm{CdSe} / \mathrm{ZnS}$ QDs in an LCORR. With the development of new materials, other $\mathrm{NCs}$ are emerging for lasing, imaging, and sensing. For example, carbon dots doped polymer achieved lasing at the visible region by coating on an optical fiber, or the $\pi$-conjugated polymer was filled in a high- $Q$ cavity to finely tune its emission spectra by mechanically stretching the resonator $[48,80]$.

\subsection{Biological gain materials}

Biological gain materials, thanks to their excellent biocompatibility, can be embedded/ implanted in cell or tissue to analyze the biological process [50, 81-84]. A series of biological gain materials have been demonstrated for lasing, including luciferin, fluorescent protein, chlorophyll, riboflavin, and indocyanine green (ICG) [35, 85-89]. The lasing mechanism of these biomaterials is similar to that of a quasi-four-level laser system shown in Fig. 5. Because the lifetime of biological gain materials in the excited state is typically a few nanoseconds, population inversion can be achieved most conveniently by optical pumping with nanosecond or shorter pulses [50].

In contrast to the dyes and NCs, which might be cytotoxic, biological gain materials are inherently biocompatible and easily biodegradable. A great variety of these materials together with their excellent optical properties, such as large transition cross-sections $\left[>2 \times 10^{-16} \mathrm{~cm}^{2}\right.$ for riboflavin and green fluorescent protein (GFP)], make them cover almost the entire visible band $[90,91]$. The sources of the biomaterials are very wide. It can be generated reproducibly in a biological system. For example, GFP is synthesized in genetically programmed Escherichia coli bacteria, mammalian cells, and jellyfish Aequorea victoria [50, 92, 93]. With fluorescent protein serving as a donor-acceptor pair, biomolecule interactions can be investigated. This group of gain medium include eGFP-eCherry, cyan fluorescent protein (CFP)-yellow fluorescent protein (YFP), and Clover-mRuby2 [86, 94]. In addition, the biomaterials can be also synthesized chemically. ICG, as the only NIR dye approved by the U.S Food and Drug Administration (FDA) for clinical use, was employed to demonstrate lasing in human serum and whole blood [89].

\section{Pump and detection}

A pump source is necessary to provide energy for the system of FOFL. It can pump the gain molecules from the low energy level to the high level and generate stimulated emission. $Q$-switched nanosecond laser and optical parametric oscillator (OPO) are the most frequently used pump source. 
The use of optical fibers in FOFL enables high coupling efficiency of the pump and remote pumping. For example, the optical fiber FP cavity $[24,26]$ and photonic bandgap fiber $[78,95,96]$ can transmit the pump laser into the optical microcavity to interact with the gain medium. The wavelength for pumping should be well-matched with the absorption band of the gain medium [Fig. 5(b)]. The pulse duration is approximately one-tenth to several times of the excited-state lifetime of the gain medium [21], otherwise the lasing properties may degrade as the excitons would go to the unintended triplet states of dyes [Fig. 5(a)] [52] or the nonradiative recombination centers/defects would generate in the case of NCs [76]. A single-pulse pump should be implemented to reduce the photobleaching effect, but on the other hand, multiple pulses can improve the output stability. In addition, the polarization of the pump laser has an impact on the emission [34, 43]. Depending on the high $Q$-factor of the optical fiber microresonator and the strong light-matter interaction, the threshold for the FOFL is often on the order of several to tens of micro Joule per square millimeter and can be as low as $15 \mathrm{~nJ} / \mathrm{mm}^{2}$ or $16 \mathrm{~nJ} /$ pulse $[42,78,85,97]$. In addition to the pulsed pump, continuous-wave (CW) lasers, electrical, and chemical pump are promising for the development of low cost and compact microlasers, but they have not been demonstrated in FOFLs. These ideas are promising and doable because of the easy integration of electrodes on optical fibers and versatile methods in the toolbox for the microfabrication of functional fiber microstructures [98, 99].

The readout of the lasing signals is very important for the analysis of laser property and the application of sensing, labeling, and imaging. The laser emission can be directionally and efficiently coupled out of the microlaser by using optical fiber. The properties of the laser emission, such as wavelength (spectrum) [51], polarization (spectrum) [34, 43], lasing mode (spectrum and imaging)
[83, 84, 100], and intensity (spectrum, imaging, and photoelectric) [22, 101], are recorded by different devices. The spectrum is the fingerprint of laser emission to reveal the intrinsic properties of the gain medium and the optical microcavity, like threshold, free spectral range (FSR), phase, and lasing modes. The laser characteristics reflected by spectra have been employed for the detection of tiny changes in the biological process [102-104]. Imaging can acquire the laser mode pattern and the spatial distribution of laser emission, which is promising to integrate with a smartphone [105]. Yun's group [50, $106,107]$ has investigated the spatial distribution of the laser transverse modes in living cell lasers by imaging. Photoelectric detection can rapidly respond to the high data throughput, which is attractive to explore in optofluidic laser.

\section{Sensing applications}

FOFLs have gained considerable interest for biochemical applications due to their high sensitivity, high signal-to-noise ratio, narrow linewidth, high reproducibility, and high integration. In this section, we review the applications of FOFLs for molecular detection and microorganism analysis.

\subsection{Biomolecular analysis}

FOFLs are sensitive to the subtle changes of gain and analyte molecules in the microresonator, because the high $Q$-factor of optical fiber microcavity can extend many times of circulation of photons in the microcavity and the nonlinear lasing process further elevates the sensitivity to the analyte. Typical optical properties of the laser, including wavelength [108], intensity [109, 110], polarization [111], and other features, can be used as sensing output to perform biomolecular detection.

Thanks to its simple setup and low cost, the intensity of FOFLs is the most frequently used sensing output to reflect the detailed information of analytes, such as concentration, RI, and molecular structure. The target can be linked to the gain medium molecules by specific binding between 
biomolecules such as biotin-streptavidin and antibody-antigen. On the other hand, the liquid gain can be directly mixed with the biomolecular in the homogeneous assay to achieve label-free biodetection. Various types of intensity-modulated FOFL biosensors have been developed and applied for high-performance biomolecular analysis, including DNA analysis $[36,112,113]$, protein detection [103], and chemicals test [114]. For example, the magnesium ionic strength induced conformational change of DNA can be detected by combining optofluidic laser with FRET [Fig. 6(a)] [115]. The conformational change of DNA Holliday junction leads to the variation of the FRET ratio,

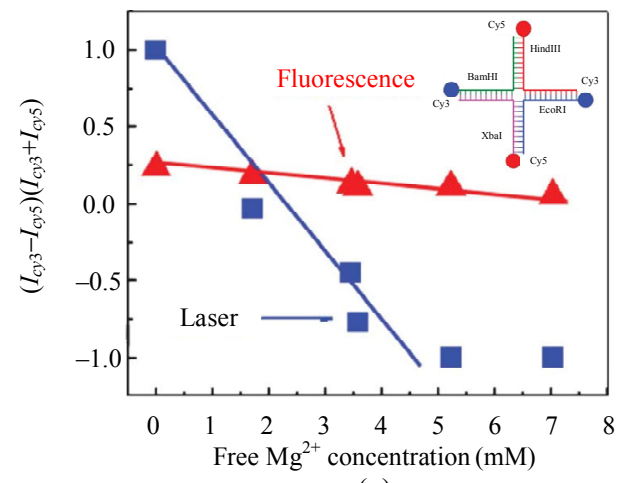

(a)

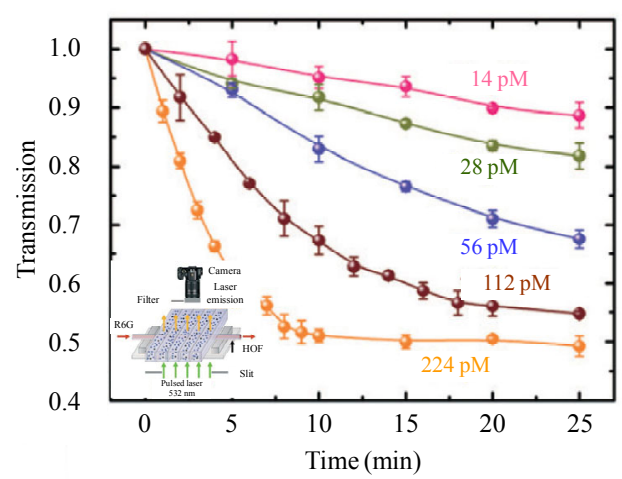

(c) which in turn corresponds to the $\mathrm{Mg}^{2+}$ concentration at a much higher sensitivity with laser-based detection than that of traditional FRET detection. Yang et al. [116] have demonstrated disposable FOFLs for protein detection [Fig. 6(b)]. The disposability is originated from the mass-production of optical fibers, enabling the optical microcavities with high reproducibility and low cost [40, 117]. Owing to the narrow linewidth of laser and the small size of optical fiber, a highly integrated array can be constructed for high throughput detection [40, 59]. Gong et al. [59] proposed a distributed FOFLs and demonstrated its high-throughput sensing in colorimetric detection [Fig. 6(c)].

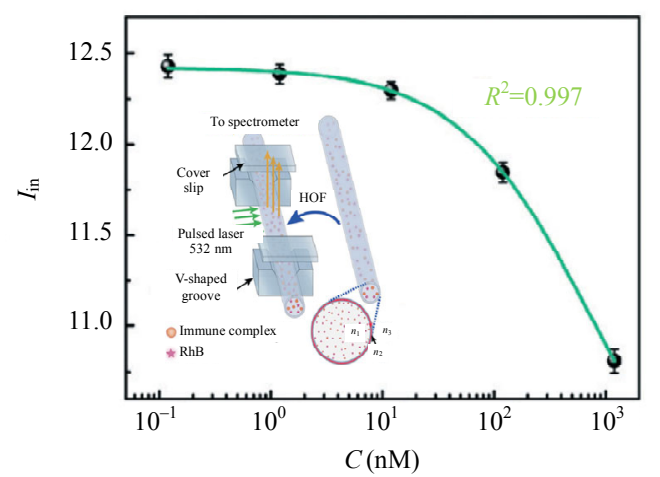

(b)

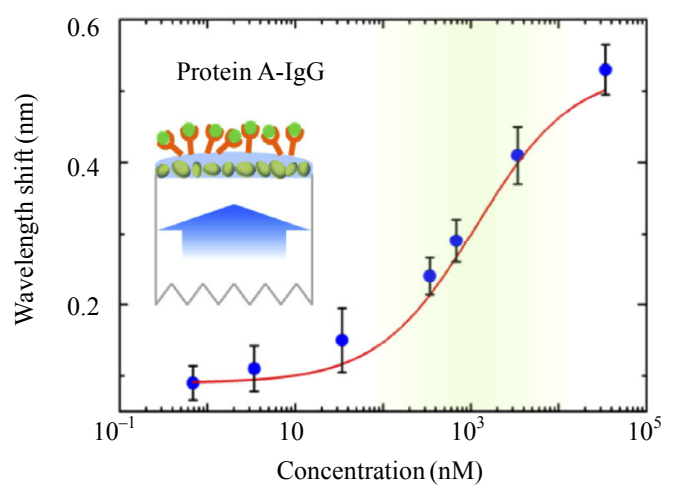

(d)

Fig. 6 FOFL for application in biomolecular analysis application of fiber optofluidic laser: (a) detection of the conformational change in a Holliday junction upon addition of $\mathrm{Mg}^{2+}$ [115], (b) disposable FOFL for the detection of protein [116], (c) array of FOFL for colorimetric detection [59], and (d) wavelength-modulated random laser with the Ag nanoparticles as scatterers [120].

Wavelength is another sensing parameter. In the passive microcavities, the RI variation of the surrounding medium alters the resonance mode, resulting in a shift of resonant peaks, mode splitting, or broadening. Similarly, for the FOFLs, the laser wavelength can also be modulated by the RI around the optical cavities. The narrow linewidth of the laser provides a high resolution for detecting the wavelength shift. Ren et al. [118] have proposed a label-free RI sensor, in which two coupled HOFs 
were finely adjusted to achieve the Verner effect to break the limit of sensitivity and reduce the detection noise. By monitoring the wavelength shift, a noise equivalent detection limit of $2.6 \times 10^{-6} \mathrm{RIU}$ was achieved [118]. It has been reported that the sensitivity can be further improved [119]. Shi et al. [120] have developed a plasmonic random laser for detecting IgG where the shift of wavelength was monitored [Fig. 6(d)].

\subsection{Cell labeling and tissue mapping}

Various microlasers have been demonstrated for labeling, tracking, and imaging of biomolecules, cells, and tissues due to their spectral multiplexing

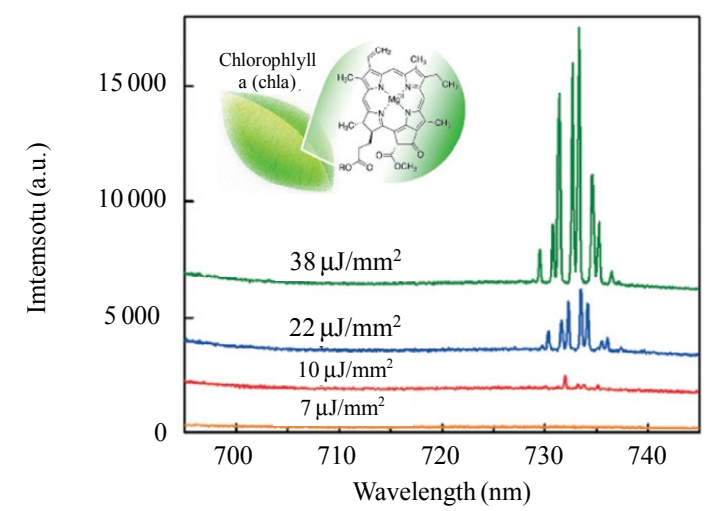

(a)

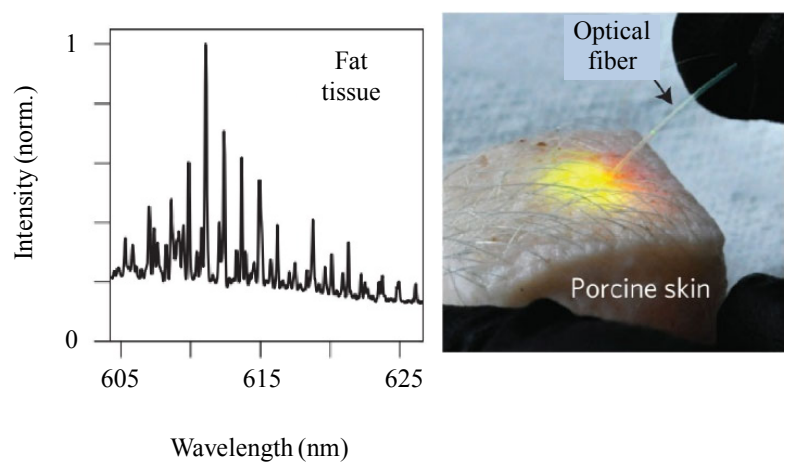

(c) capability, strong background suppression, and high contrast ratio [50, 51, 105]. Optofluidic lasers have also been widely applied in investigating the interaction between proteins [86], exploring the metabolic functions and biostructure in living organisms [121], and screening the tumor tissues visually $[105,122]$. The microlasers in cell, blood, and tissue can be labeled with intrinsic (fluorescent proteins $[50,81,82,84,123])$ or exogenous (dyes [106, 107]) gain materials and devised using conventional (microbeads [83], liquid droplets [124], and FP cavity [125]) or derived (random scattering $[126,127])$ optical microresonators.

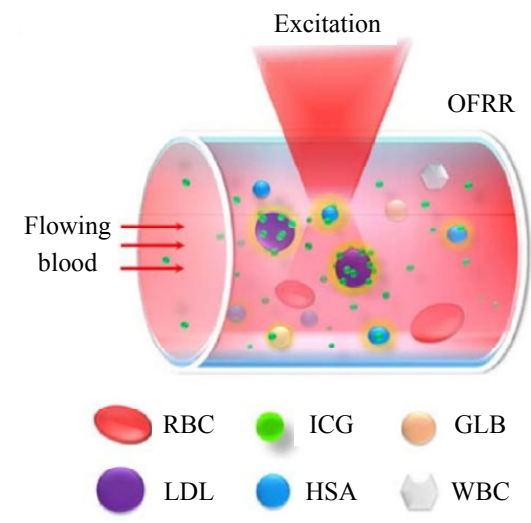

(b)

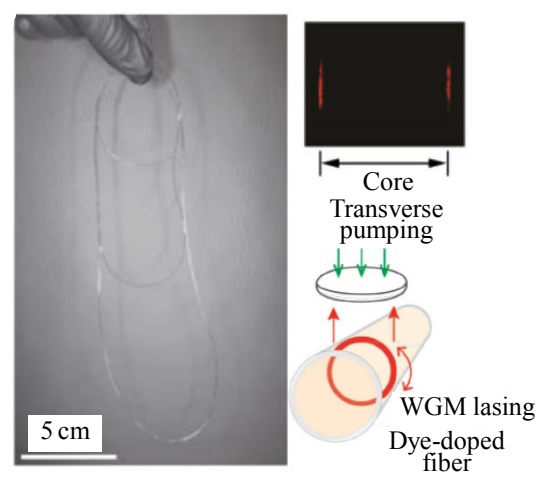

(d)

Fig. 7 Lasing in blood and tissue: (a) chlorophyll laser with a high $Q$-factor optofluidic ring resonator [87], (b) lasing in human whole blood by using ICG to detect lipoproteins and albumins [89], (c) cellular laser from fat tissue by inserting an optical fiber into the subcutaneous fat layer to guide the pump laser and collect the laser emission [83], and (d) a biocompatible hydrogel optical fiber for WGM lasing [129].

Initially, cell lasers were achieved by sandwiching the cell in an external FP cavity, which is more versatile and can be applied for any type of cell. Chen et al. [125] have integrated a microwell 
array within the FP cavity for addressable and automated cell laser. To be implantable, the microlasers with micrometer sizes were embedded inside the living cells to achieve massive tagging and subsequent tracking of cells. Yun's group [51] investigated the real-time tracking of thousands of individual cells by implanting the laser particles in the cells. The spatial information of tissue (collection of cells) can also be mapped by laser emissions, such as cancerous tissue $[105,122]$ and bone tissue [128]. Chen et al. [105] has demonstrated the mapping of cancer tissue by laser-based imaging, which has the potential for cancer screening and immunodiagnosis. The imaging has a subcellular and sub-micrometer resolution.

The diversity in gain material and structures of microresonators makes many kinds of FOFLs possible. The biocompatible materials are crucial for bio-applications of cells and tissues. Chen et al. [87] have developed a chlorophyll laser shown in Fig. 7(a). The most abundant pigment in the plant chlorophyll a (Chla) - was applied in the FOFLs as a gain medium to investigate its lasing mechanism and it has shown dual lasing bands at around $680 \mathrm{~nm}$ and $730 \mathrm{~nm}$. The chlorophyll laser provides an important insight into the photosynthesis process inside plants. Chen et al. [89] demonstrated a blood laser by injecting the ICG into the human serum and whole blood at clinically acceptable concentrations [Fig. 7(b)]. Optical fiber is an ideal candidate for developing endoscopes and performing surgery with reduced invasive injury, thanks to its high aspect ratio and high optical transparency. Figure 7(c) illustrates a cellular laser in tissue, in which an optical fiber is inserted into the subcutaneous fat layer to optically pump adipocytes to collect the lasing emission from the tissue [83]. Besides, the optical fiber can be fabricated with biocompatible and biodegradable materials, enabling the possibility of implanting optical fiber into the tissue for mapping. For example, the high flexibility of hydrogel optical fibers allows them to be easily implanted into tissue. Choi et al. [129] have observed a light amplification in the tangential direction of core-clad step-index hydrogel optical fiber through WGM lasing [Fig. 7(d)].

\section{Perspectives}

Fiber optofluidic lasers have become a hot research topic in recent years. They have been developed as both a new category of tunable laser devices and a high performance sensing technology. In the next decade, the FOFLs will be undoubtedly developing quickly due to the innovations in materials and fiber designs, as well as the utilization of advanced fabrication technologies.

The laser emission depends strongly on the material properties. For example, a full-color laser can be developed by combining the reconfigurability of microfluidics and the diversity of gain material, such as the highly photostable organic semiconductors, upconversion nanoparticles, and conjugated polymers [130-132]. In addition, the biocompatible and soft polymers can be easily fabricated to form an optical microcavity implantable into a tissue for in situ monitoring [129, 133]. Saturable absorber materials might be integrated with FOFLs to achieve ultrafast laser, through $Q$-switching or mode-locking, for monitoring ultrafast biochemical reactions $[134,135]$.

Thanks to the structural flexibility of optical fibers (such as size, microstructure, and length), novel applications of FOFLs become highly expected, such as high-throughput bioassay. The advanced micromachining technologies enable various microstructures to be inscribed inside the optical fiber for achieving high integration and multi-functionality [136]. The microstructures can also be mass-produced with high repeatability and low cost by designing the fiber preform and the fiber drawing process [137]. Owing to the large aspect ratio of optical fiber, the lab-on-fiber tip biolaser is hopeful to achieve remotely space-constrained 
imaging like endoscope and sub-diffraction limit imaging in the far-field [138].

\section{Conclusions}

Fiber optofluidic laser possesses a set of unique advantages related to both optical fiber (high repeatability, low cost, large aspect ratio, and high integration) and laser (optical feedback, threshold behavior, narrow linewidth, and rich spectral characteristics), which make them serve as a promising platform for high-performance biochemical sensing. This review has summarized the recent progress of fiber optofluidic lasers, including the optical fiber microcavities, gain materials, and pump and detection methods for this novel laser. The applications of FOFLs in biomolecular analysis, cell labeling, and tissue mapping have also been highlighted. The prospects of further development and research directions are also discussed. We believe that the innovations in gain material and biomaterials, as well as the novel design of optical fiber microresonators will continuously push forward the fiber optofluidic lasers and their applications.

\section{Acknowledgment}

The authors gratefully acknowledge the financial support from the National Natural Science Foundation of China (Grant No. 61875034), the 111 Project (Grant No. B14039), and Seeding Project of Scientific and Technical Innovation of Sichuan Province (Grant No. 2020107).

Open Access This article is distributed under the terms of the Creative Commons Attribution 4.0 International License (http://creativecommons.org/licenses/by/4.0/), which permits unrestricted use, distribution, and reproduction in any medium, provided you give appropriate credit to the original author(s) and the source, provide a link to the Creative Commons license, and indicate if changes were made.

\section{References}

[1] F. T. S. Yu and S. Yin, Fiber optic sensors. New
York: Wiley Encyclopedia of Biomedical Engineering, 2002.

[2] M. Janik, E. Brzozowska, P. Czyszczon, A. Celebanska, M. Koba, and A. Gamian, “Optical fiber aptasensor for label-free bacteria detection in small volumes," Sensors and Actuators B, 2020, 330: 129316.

[3] M. Consales, M. Pisco, and A. Cusano, "Lab-on-fiber technology: a new avenue for optical nanosensors," Photonic Sensors, 2012, 2(4): 289-314.

[4] H. C. Lefevre, The fiber-optic gyroscope. London: Artech House, 2014.

[5] Y. J. Rao, "Recent progress in applications of in-fibre Bragg grating sensors," Optics and Lasers in Engineering, 1999, 31(4): 297-324.

[6] A. P. Zhang, S. Gao, G. Yan, and Y. Bai, "Advances in optical fiber bragg grating sensor technologies," Photonic Sensors, 2012, 2(1): 1-13.

[7] A. D. Kersey, M. A. Davis, H. J. Patrick, M. LeBlanc, K. P. Koo, C. G. Askins, et al., "Fiber grating sensors," Journal of Lightwave Technology, 1997, 15(8): 1442-1462.

[8] G. Yu, Z. Cai, Y. Chen, X. Wang, Q. Zhang, Y. Li, et al., "Walkaway VSP using multimode optical fibres in a hybrid wireline," The Leading Edge, 2016, 35(7): 936-940.

[9] E. F. Williams, M. R. Fernández-Ruiz, R. Magalhaes, R. Vanthillo, Z. Zhan, M. González-Herráez, et al., "Distributed sensing of microseisms and teleseisms with submarine dark fibers," Nature Communications, 2019, 10: 5778.

[10] A. Barrias, J. R. Casas, and S. Villalba, "A review of distributed optical fiber sensors for civil engineering applications," Sensors, 2016, 16(5): 748.

[11] H. Murayama, D. Wada, and H. Igawa, "Structural health monitoring by using fiber-optic distributed strain sensors with high spatial resolution," Photonic Sensors, 2013, 3(4): 355-376.

[12] A. Leung, P. M. Shankar, and R. Mutharasan, "A review of fiber-optic biosensors," Sensors and Actuators B, 2007, 125(2): 688-703.

[13] A. B. Socorro-Leránoz, D. Santano, I. Del Villar, and I. R. Matias, "Trends in the design of wavelength-based optical fibre biosensors (2008-2018)," Biosensors and Bioelectronics, 2019, 1: 100015.

[14] X. D. Wang and O. S. Wolfbeis, "Fiber-optic chemical sensors and biosensors (2015-2019)," Analytical Chemistry, 2020, 92(1): 397-430.

[15] M. Piestrzynska, M. Dominik, K. Kosiel, M. Janczuk-Richter, K. Szot-Karpinska, and E. Brzozowska, "Ultrasensitive tantalum oxide nano-coated long-period gratings for detection of various biological targets," Biosensors and Bioelectronics, 2019, 133: 8-15.

[16] Y. Zhao, X. G Hu, S. Hu, and Y. Peng, 
"Applications of fiber-optic biochemical sensor in microfluidic chips: a review," Biosensors and Bioelectronics, 2020, 166: 112447.

[17] L. Zhang, J. Lou, and L. Tong, "Micro/nanofiber optical sensors," Photonic Sensors, 2011, 1(1): 31-42.

[18] Y. Wang, S. Meng, Y. Liang, L. Li, and W. Peng, "Fiber-optic surface plasmon resonance sensor with multi-alternating metal layers for biological measurement," Photonic Sensors, 2013, 3(3): 202-207.

[19] A. P. Demchenko, Introduction to fluorescence sensing. Ukraine: Springer Science \& Business Media, 2008.

[20] Y. C. Chen and X. Fan, "Biological lasers for biomedical applications," Advanced Optical Materials, 2019, 7(17): 1900377.

[21] X. Fan and S. H. Yun, "The potential of optofluidic biolasers," Nature Methods, 2014, 11(2): 141-147.

[22] X. Wu, M. K. K. Oo, K. Reddy, Q. Chen, Y. Sun, and X. Fan, "Optofluidic laser for dual-mode sensitive biomolecular detection with a large dynamic range," Nature Communications, 2014, 5: 3779 .

[23] H. Zhou, G. Feng, K. Yao, C. Yang, J. Yi, and S. Zhou, "Fiber-based tunable microcavity fluidic dye laser," Optics Letters, 2013, 38(18): 3604-3607.

[24] Q. Kou, I. Yesilyurt, and Y. Chen, "Collinear dual-color laser emission from a microfluidic dye laser," Applied Physics Letters, 2006, 88(9): 091101.

[25] G. Aubry, Q. Kou, J. Soto-Velasco, C. Wang, S. Meance, J. J. He, et al., "A multicolor microfluidic droplet dye laser with single mode emission," Applied Physics Letters, 2011, 98(11): 111111.

[26] R. M. Gerosa, A. Sudirman, L. de S. Menezes, W. Margulis, and C. J. S. de Matos, "All-fiber high repetition rate microfluidic dye laser," Optica, 2015, 2(2): 186-193.

[27] C. Gong, Y. Gong, W. L. Zhang, Y. Wu, Y. J. Rao, G. D. Peng, et al., "Fiber optofluidic microlaser with lateral single mode emission," IEEE Journal of Selected Topics in Quantum Electronics, 2018, 24(3): 0900206.

[28] F. Vollmer and S. Arnold, "Whispering-gallerymode biosensing: label-free detection down to single molecules," Nature Methods, 2008, 5(7): 591-596.

[29] M. G. Suh, Q. F. Yang, K. Y. Yang, X. Yi, and K. J. Vahala, "Microresonator soliton dual-comb spectroscopy,” Sicence, 2016, 354(6312): 600-603.

[30] Y. Zhi, X. C. Yu, Q. Gong, L. Yang, and Y. F. Xiao, "Single nanoparticle detection using optical microcavities," Advanced Materials, 2017, 29(12): 1604920.

[31] H. J. Moon, Y. T. Chough, and K. An, "Cylindrical microcavity laser based on the evanescent-wavecoupled gain," Physical Review Letters, 2000, 85(15): 3161-3164.
[32] Y. Sun, J. D. Suter, and X. Fan, "Robust integrated optofluidic-ring-resonator dye lasers," Optics Letters, 2009, 34(7): 1042-1044.

[33] X. Wu, Y. Sun, J. D. Suter, and X. Fan, "Single mode coupled optofluidic ring resonator dye lasers," Applied Physics Letters, 2009, 94(24): 241109.

[34] Y. Zhang, W. Meng, H. Yang, Y. Chu, and X. Pu, "Demonstration of polarization mode selection and coupling efficiency of optofluidic ring resonator lasers," Optics Letters, 2015, 40(21): 5101-5104.

[35] Q. Chen, M. Ritt, S. Sivaramakrishnan, Y. Sun, and $\mathrm{X}$. Fan, "Optofluidic lasers with a single molecular layer of gain," Lab on a Chip, 2014, 14(24): 4590-4595.

[36] W. Lee, Q. Chen, X. Fan, and D. K. Yoon, "Digital DNA detection based on a compact optofluidic laser with ultra-low sample consumption," Lab on a Chip, 2016, 16(24): 4770-4776.

[37] S. I. Shopova, H. Zhou, X. Fan, and P. Zhang, "Optofluidic ring resonator based dye laser," Applied Physics Letters, 2007, 90(22): 221101.

[38] S. Lacey, I. M. White, Y. Sun, S. I. Shopova, J. M. Cupps, P. Zhang, et al., "Versatile opto-fluidic ring resonator lasers with ultra-low threshold," Optics Express, 2007, 15(23): 15523-15530.

[39] Y. Wang, K. S. Leck, D. Van Ta, R. Chen, V. Nalla, Y. Gao, et al., "Blue liquid lasers from solution of $\mathrm{CdZnS} / \mathrm{ZnS}$ ternary alloy quantum dots with quasi-continuous pumping," Advanced Materials, 2015, 27(1): 169-175.

[40] Y. Xu, C. Gong, Q. Chen, Y. Luo, Y. Wu, Y. Wang, et al., "Highly reproducible, isotropic optofluidic laser based on hollow optical fiber," IEEE Journal of Selected Topics in Quantum Electronics, 2019, 25(1): 0900206.

[41] D. Yan, H. Zhang, B. Liu, B. Huang, J. Sun, and D. Wang, "Optofluidic microring resonator laser based on cavity-assisted energy transfer in dye-infiltrated side-hole microstructured optical fibers," Journal of Lightwave Technology, 2017, 35(19): 4153-4158.

[42] J. Yu, Y. Liu, Y. Wang, Z. Wang, X. Zhang, X. Liu, et al., "Optofluidic laser based on a hollow-core negative-curvature fiber," Nanophotonics, 2018, 7(7): 1307-1315.

[43] D. Yan, B. Liu, H. Zhang, Y. Li, and T. Han, "Observation of lasing emission based on a hexagonal cavity embedded in a Kagome PCF," IEEE Photonics Technology Letters, 2018, 30(13): 1202-1205.

[44] Z. L. Li, Y. G. Liu, M. Yan, W. Y. Zhou, C. F. Ying, Q. Ye, et al., "A simplified hollow-core microstructured optical fibre laser with microring resonators and strong radial emission," Applied Physics Letters, 2014, 105(7): 071902.

[45] B. H. Soffer and B. B. McFarland, "Continuously tunable, narrow-band organic dye lasers," Applied Physics Letters, 1967, 10(10): 266-267. 
[46] A. Jha, B. Richards, G. Jose, T. Teddy-Fernandez, P. Joshi, X. Jiang, et al., "Rare-earth ion doped $\mathrm{TeO}_{2}$ and $\mathrm{GeO}_{2}$ glasses as laser materials," Progress in Materials Science, 2012, 57(8): 1426-1491.

[47] V. I. Klimov, A. A. Mikhailovsky, S. Xu, A. Malko, J. A. Hollingsworth, C. A. Leatherdale, et al., "Optical gain and stimulated emission in nanocrystal quantum dots," Science, 2000, 290(5490): 314-317.

[48] W. F. Zhang, H. Zhu, S. F. Yu, and H. Y. Yang, "Observation of lasing emission from carbon nanodots in organic solvents," Advanced Materials, 2012, 24(17): 2263-2267.

[49] R. G. Smart, D. C. Hanna, A. C. Tropper, S. T. Davey, S. F. Carter, and D. Szebesta, "CW room temperature upconversion lasing at blue, green and red wavelengths in infrared-pumped $\mathrm{Pr}^{3+}$-doped fluoride fibre," Electronics Letters, 1991, 27(14): 1307-1309.

[50] M. C. Gather and S. H. Yun, "Single-cell biological lasers," Nature Photonics, 2011, 5(7): 406-410.

[51] N. Martino, S. J. J. Kwok, A. C. Liapis, S. Forward, H. Jang, H. M. Kim, et al., "Wavelength-encoded laser particles for massively multiplexed cell tagging," Nature Photonics, 2019, 13(10): 720-727.

[52] Z. Li and D. Psaltis, "Optofluidic dye lasers," Microfluidics and Nanofluidics, 2008, 4(1-2): 145-158.

[53] J. Wu, W. Wang, C. Gong, Q. Li, Z. Li, G. Deng, et al., "Tuning the strength of intramolecular charge-transfer of triene-based nonlinear optical dyes for electro-optics and optofluidic lasers," Journal of Materials Chemistry C, 2017, 5(30): $7472-7478$.

[54] Y. Chen, L. Lei, K. Zhang, J. Shi, L. Wang, H. Li, et al., "Optofluidic microcavities: dye-lasers and biosensors," Biomicrofluidics, 2010, 4(4): 043002.

[55] W. Lee, Y. Sun, H. Li, K. Reddy, M. Sumetsky, and $\mathrm{X}$. Fan, "A quasi-droplet optofluidic ring resonator laser using a micro-bubble," Applied Physics Letters, 2011, 99(9): 091102.

[56] J. D. Suter, W. Lee, D. J. Howard, E. Hoppmann, I. M. White, and X. Fan, "Demonstration of the coupling of optofluidic ring resonator lasers with liquid waveguides," Optics Letters, 2010, 35(17): 2997-2999.

[57] H. J. Moon, Y. T. Chough, J. B. Kim, K. An, J. Yi, and $\mathrm{J}$. Lee, "Cavity- $Q$-driven spectral shift in a cylindrical whispering-gallery-mode microcavity laser," Applied Physics Letters, 2000, 76(25): 3679-3681.

[58] H. J. Moon, J. Yi, J. T. Kim, and J. Lee, "Effect of refractive index change on the interference modulation of $Q$ values in a layered cylindrical microlaser," Japanese Journal of Applied Physics, 1999, 38(4): L377-L379.

[59] C. Gong, Y. Gong, X. Zhao, Y. Luo, Q. Chen, $\mathrm{X}$. Tan, et al., "Distributed fibre optofluidic laser for chip-scale arrayed biochemical sensing," Lab on a Chip, 2018, 18(18): 2741-2748.

[60] S. V. Frolov, M. Shkunov, Z. V. Vardeny, and K. Yoshino, "Ring microlasers from conducting polymers," Physical Review B, 1997, 56(8): R4363-R4366.

[61] X. Zhao, Y. Wang, C. Liao, G. D. Peng, Y. Gong, and Y. Wang, "Polymer-coated hollow fiber optofluidic laser for refractive index sensing," Journal of Lightwave Technology, 2020, 38(6): 1550-1556.

[62] H. Tanaka, Y. Yoshida, T. Nakao, N. Tsujimoto, A. Fujii, and M. Ozaki, "Photopumped laser oscillation and charge carrier mobility of composite films based on poly(3-hexylthiophene)s with different stereoregularity," Japanese Journal of Applied Physics, 2006, 45(37-41): L1077-L1079.

[63] Y. Yoshida, Y. Nishihara, A. Fujii, M. Ozaki, K. Yoshino, H. K. Kim, et al., "Optical properties and microring laser of conducting polymers with $\mathrm{Sn}$ atoms in main chains," Journal of Applied Physics, 2004, 95(8): 4193-4196.

[64] Y. Yoshida, T. Nishimura, A. Fujii, M. Ozaki, and K. Yoshino, "Dual ring laser emission of conducting polymers in microcapillary structures," Applied Physics Letters, 2005, 86(14): 141903.

[65] H. Yanagi, R. Takeaki, S. Tomita, A. Ishizumi, F. Sasaki, K. Yamashita, et al., "Dye-doped polymer microring laser coupled with stimulated resonant Raman scattering," Applied Physics Letters, 2009, 95(3): 033306.

[66] H. J. Moon, G. W. Park, S. B. Lee, K. An, and J. H. Lee, "Laser oscillations of resonance modes in a thin gain-doped ring-type cylindrical microcavity," Optics Communications, 2004, 235(4-6): 401-407.

[67] A. François, N. Riesen, K. Gardner, T. M. Monro, and A. Meldrum, "Lasing of whispering gallery modes in optofluidic microcapillaries," Optics Express, 2016, 24(12): 12466-12477.

[68] A. P. Alivisatos, "Semiconductor clusters, nanocrystals, and quantum dots," Science, 1996, 271(5251): 933-937.

[69] M. Bruchez, M. Moronne, P. Gin, S. Weiss, and A. P. Alivisatos, "Semiconductor nanocrystals as fluorescent biological labels," Science, 1998, 281(5385): 2013-2016.

[70] U. Resch-Genger, M. Grabolle, S. Cavaliere-Jaricot, R. Nitschke, and T. Nann, "Quantum dots versus organic dyes as fluorescent labels," Nature Methods, 2008, 5(9): 763-775.

[71] L. E. Brus, "Electron-electron and electron-hole interactions in small semiconductor crystallites: The size dependence of the lowest excited electronic state," The Journal of Chemical Physics, 1984, 80(9): 4403-4409.

[72] M. Han, X. Gao, J. Z. Su, and S. Nie, "Quantum-dot-tagged microbeads for multiplexed 
optical coding of biomolecules," Nature Biotechnology, 2001, 19(7): 631-635.

[73] P. Alivisatos, "The use of nanocrystals in biological detection," Nature Biotechnology, 2004, 22(1): 47-52.

[74] V. I. Klimov, S. A. Ivanov, J. Nanda, M. Achermann, I. Bezel, J. A. McGuire, et al., "Single-exciton optical gain in semiconductor nanocrystals," Nature, 2007, 447(7143): 441-446.

[75] J. Schafer, J. P. Mondia, R. Sharma, Z. H. Lu, A. S. Susha, A. L. Rogach, et al., "Quantum dot microdrop laser," Nano Letters, 2008, 8(6): 1709-1712.

[76] Y. Wang, K. S. Leck, V. D. Ta, R. Chen, V. Nalla, Y. Gao, et al., "Blue liquid lasers from solution of $\mathrm{CdZnS/ZnS} \mathrm{ternary} \mathrm{alloy} \mathrm{quantum} \mathrm{dots} \mathrm{with}$ quasi-continuous pumping," Advanced Materials, 2015, 27(1): 169-175.

[77] M. Kazes, D. Y. Lewis, Y. Ebenstein, T. Mokari, and U. Banin, "Lasing from semiconductor quantum rods in a cylindrical microcavity," Advanced Materials, 2002, 14(4): 317-321.

[78] N. Zhang, H. Liu, A. M. Stolyarov, T. Zhang, K. Li, P. P. Shum, et al., "Azimuthally polarized radial emission from a quantum dot fiber laser," $A C S$ Photonics, 2016, 3(12): 2275-2279.

[79] A. Kiraz, Q. Chen, and X. Fan, "Optofluidic lasers with aqueous quantum dots," ACS Photonics, 2015, 2(6): 707-713.

[80] S. J. Tang, Z. Liu, Y. J. Qian, K. Shi, Y. Sun, C. Wu, et al., "A tunable optofluidic microlaser in a photostable conjugated polymer," Advanced Materials, 2018, 30(50): 1804556.

[81] S. Nizamoglu, M. C. Gather, and S. H. Yun, "All-biomaterial laser using vitamin and biopolymers," Advanced Materials, 2013, 25(41): 5943-5947.

[82] A. Jonáš, M. Aas, Y. Karadag, S. Manioğlu, S. Anand, D. McGloin, et al., "In vitro and in vivo biolasing of fluorescent proteins suspended in liquid microdroplet cavities," Lab on a Chip, 2014, 14(16): 3093-3100.

[83] M. Humar and S. H. Yun, "Intracellular microlasers," Nature Photonics, 2015, 9(9): 572-577.

[84] M. Humar and S. H. Yun, "Whispering-gallerymode emission from biological luminescent protein microcavity assemblies," Optica, 2017, 4(2): 222-228.

[85] X. Wu, Q. Chen, Y. Sun, and X. Fan, "Bio-inspired optofluidic lasers with luciferin," Applied Physics Letters, 2013, 102(20): 203706.

[86] Q. Chen, X. Zhang, Y. Sun, M. Ritt, S. Sivaramakrishnan, and X. Fan, "Highly sensitive fluorescent protein FRET detection using optofluidic lasers," Lab on a Chip, 2013, 13(14): 2679-2681.

[87] Y. C. Chen, Q. Chen, and X. Fan, "Optofluidic chlorophyll lasers," Lab on a Chip, 2016, 16(12): 2228-2235.

[88] W. Lee, D. Bin Kim, M. H. Song, and D. K. Yoon, "Optofluidic ring resonator laser with an edible liquid gain medium," Optics Express, 2017, 25(13): 14043-14048.

[89] Y. C. Chen, Q. Chen, and X. Fan, "Lasing in blood," Optica, 2016, 3(8): 809-815.

[90] N. C. Shaner, R. E. Campbell, P. A. Steinbach, B. N. G. Giepmans, A. E. Palmer, and R. Y. Tsien, "Improved monomeric red, orange and yellow fluorescent proteins derived from Discosoma sp. red fluorescent protein," Nature Biotechnology, 2004, 22(12): 1567-1572.

[91] E. M. Merzlyak, J. Goedhart, D. Shcherbo, M. E. Bulina, A. S. Shcheglov, A. F. Fradkov, et al., "Bright monomeric red fluorescent protein with an extended fluorescence lifetime," Nature Methods, 2007, 4(7): 555-557.

[92] M. C. Gather and S. H. Yun, "Lasing from Escherichia coli bacteria genetically programmed to express green fluorescent protein," Optics Letters, 2011, 36(16): 3299-3301.

[93] R. Y. Tsien and A. Miyawaki, "Seeing the machinery of live cells," Science, 1998, 280(5371): 1954-1955.

[94]A. J. Lam, F. St-Pierre, Y. Gong, J. D. Marshall, P. J. Cranfill, M. A. Baird, et al., "Improving FRET dynamic range with bright green and red fluorescent proteins," Nature Methods, 2012, 9(10): 1005-1012.

[95] A. M. Stolyarov, L. Wei, O. Shapira, F. Sorin, S. L. Chua, J. D. Joannopoulos, et al., "Microfluidic directional emission control of an azimuthally polarized radial fibre laser," Nature Photonics, 2012, 6(4): 229-233.

[96] O. Shapira, K. Kuriki, N. D. Orf, A. F. Abouraddy, G. Benoit, F. Viens, et al., "Surface-emitting fiber lasers," Optics Express, 2006, 14(9): 3929-3935.

[97] Z. L. Li, W. Y. Zhou, M. M. Luo, Y. G. Liu, and J. G. Tian, "Tunable optofluidic microring laser based on a tapered hollow core microstructured optical fiber," Optics Express, 2015, 23(8): 10413-10420.

[98] M. Rein, V. D. Favrod, C. Hou, T. Khudiyev, A. Stolyarov, J. Cox, et al., "Diode fibres for fabric-based optical communications," Nature, 2018, 560(7717): 214-218.

[99] S. Park, Y. Guo, X. Jia, H. K. Choe, B. Grena, J. Kang, et al., "One-step optogenetics with multifunctional flexible polymer fibers," Nature Neuroscience, 2017, 20(4): 612-619.

[100] A. Fernandez-Bravo, K. Yao, E. S. Barnard, N. J. Borys, E. S. Levy, B. Tian, et al., "Continuouswave upconverting nanoparticle microlasers," Nature Nanotechnology, 2018, 13(7): 572-577.

[101] M. Schubert, A. Steude, P. Liehm, N. M. Kronenberg, M. Karl, E. C. Campbell, et al., 
"Lasing within live cells containing intracellular optical microresonators for barcode-type cell tagging and tracking," Nano Letters, 2015, 15(8): 5647-5652.

[102] C. Gong, Y. Gong, M. K. Khaing Oo, Y. Wu, Y. Rao, X. Tan, et al., "Sensitive sulfide ion detection by optofluidic catalytic laser using horseradish peroxidase (HRP) enzyme," Biosensors and Bioelectronics, 2017, 96: 351-357.

[103] H. Li, L. Shang, X. Tu, L. Liu, and L. Xu, "Coupling variation induced ultrasensitive label-free biosensing by using single mode coupled microcavity laser," Journal of the American Chemical Society, 2009, 131(46): 16612-16613.

[104] Y. Sun, S. I. Shopova, C. S. Wu, S. Arnold, and X. Fan, "Bioinspired optofluidic FRET lasers via DNA scaffolds," Proceedings of the National Academy of Sciences of the United States of America, 2010, 107(37): 16039-16042.

[105] Y. C. Chen, X. Tan, Q. Sun, Q. Chen, W. Wang, and $\mathrm{X}$. Fan, "Laser-emission imaging of nuclear biomarkers for high-contrast cancer screening and immunodiagnosis," Nature Biomedical Engineering, 2017, 1(9): 724-735.

[106] S. Nizamoglu, K. B. Lee, M. C. Gather, K. S. Kim, M. Jeon, S. Kim, et al., "A simple approach to biological single-cell lasers via intracellular dyes," Advanced Optical Materials, 2015, 3(9): 1197-1200.

[107] M. Humar, M. C. Gather, and S. H. Yun, "Cellular dye lasers: lasing thresholds and sensing in a planar resonator," Optics Express, 2015, 23(21): 27865-27879.

[108] L. Ren, X. Wu, M. Li, X. Zhang, L. Liu, and L. Xu, "Ultrasensitive label-free coupled optofluidic ring laser sensor," Optics Letters, 2012, 37(18): 3873-3875.

[109] Q. Chen, H. Liu, W. Lee, Y. Sun, D. Zhu, H. Pei, et al., "Self-assembled DNA tetrahedral optofluidic lasers with precise and tunable gain control," $L a b$ on a Chip, 2013, 13(17): 3351-3354.

[110] Y. Sun and X. Fan, "Highly selective single-nucleotide polymorphism detection with optofluidic ring resonator lasers," in Conference on Lasers and Electro-Optics: Science and Innovations, California, 2011, pp. CWL6.

[111] Z. Yuan, X. Cheng, Y. Zhou, X. Tan, X. Gong, H. Rivy, et al., "Distinguishing small molecules in microcavity with molecular laser polarization," ACS Photonics, 2020, 7(8): 1908-1914.

[112] W. Lee and X. Fan, "Intracavity DNA melting analysis with optofluidic lasers," Analytical Chemistry, 2012, 84(21): 9558-9563.

[113] Y. Sun and X. Fan, "Distinguishing DNA by analog-to-digital-like conversion by using optofluidic lasers," Angewandte Chemie International Edition, 2012, 51(5): 1236-1239.
[114] J. C. Galas, C. Peroz, Q. Kou, and Y. Chen, "Microfluidic dye laser intracavity absorption," Applied Physics Letters, 2006, 89(22): 224101.

[115] X. Zhang, W. Lee, and X. Fan, "Bio-switchable optofluidic lasers based on DNA Holliday junctions," Lab on a Chip, 2012, 12(19): 3673-3675.

[116] X. Yang, Y. Luo, Y. Liu, C. Gong, Y. Wang, Y. J. Rao, et al., "Mass production of thin-walled hollow optical fibers enables disposable optofluidic laser immunosensors," Lab on a Chip, 2020, 20(5): 923-930.

[117] C. Gong, Y. Gong, Q. Chen, Y. J. Rao, G. D. Peng, and X. Fan, "Reproducible fiber optofluidic laser for disposable and array applications," Lab on a Chip, 2017, 17(20): 3431-3436.

[118] L. Ren, X. Zhang, X. Guo, H. Wang, and X. Wu, "High-sensitivity optofluidic sensor based on coupled liquid-core laser," IEEE Photonics Technology Letters, 2017, 29(8): 639-642.

[119] X. Zhang, L. Ren, X. Wu, H. Li, L. Liu, and L. Xu, "Coupled optofluidic ring laser for ultrahighsensitive sensing," Optics Express, 2011, 19(22): 22242-22247.

[120] X. Shi, K. Ge, J. H. Tong, and T. Zhai, "Low-cost biosensors based on a plasmonic random laser on fiber facet," Optics Express, 2020, 28(8): 12233-12242.

[121] Y. Wei, X. Lin, C. Wei, W. Zhang, Y. Yan, and Y. S. Zhao, "Starch-based biological microlasers," ACS Nano, 2017, 11(1): 597-602.

[122] Y. C. Chen, Q. Chen, T. Zhang, W. Wang, and $\mathrm{X}$. Fan, "Versatile tissue lasers based on high- $Q$ Fabry-Pérot microcavities," Lab on a Chip, 2017, 17(3): 538-548.

[123] C. Vannahme, F. Maier-Flaig, U. Lemmer, and A. Kristensen, "Single-mode biological distributed feedback laser," Lab on a Chip, 2013, 13(14): 2675-2678.

[124] M. Humar, A. Dobravec, X. Zhao, and S. H. Yun, "Biomaterial microlasers implantable in the cornea, skin, and blood," Optica, 2017, 4(9): 1080-1085.

[125] Q. Chen, Y. C. Chen, Z. Zhang, B. Wu, R. Coleman, and X. Fan, "An integrated microwell array platform for cell lasing analysis," Lab on a Chip, 2017, 17(16): 2814-2820.

[126] R. C. Polson and Z. V. Vardeny, "Random lasing in human tissues," Applied Physics Letters, 2004, 85(7): 1289-1291.

[127] R. C. Polson and Z. V. Vardeny, "Cancerous tissue mapping from random lasing emission spectra," Journal of Optics, 2010, 12(2): 024010.

[128]Q. Song, S. Xiao, Z. Xu, J. Liu, X. Sun, V. Drachev, et al., "Random lasing in bone tissue," Optics Letters, 2010, 35(9): 1425-1427.

[129] M. Choi, M. Humar, S. Kim, and S. H. Yun, "Step-index optical fiber made of biocompatible 
hydrogels," Advanced Materials, 2015, 27(27): 4081-4086.

[130] J. Zhao, Y. Yan, Z. Gao, Y. Du, H. Dong, J. Yao, et al., "Full-color laser displays based on organic printed microlaser arrays," Nature Communications, 2019, 10: 870.

[131] X. Chen, L. Jin, W. Kong, T. Sun, W. Zhang, $\mathrm{X}$. Liu, et al., "Confining energy migration in upconversion nanoparticles towards deep ultraviolet lasing," Nature Communications, 2016, 7: 10304.

[132] M. Karl, J. M. E. Glackin, M. Schubert, N. M. Kronenberg, G. A. Turnbull, I. D. W. Samuel, et al., "Flexible and ultra-lightweight polymer membrane lasers," Nature Communications, 2018, 9: 1525.

[133] Y. J. Heo, H. Shibata, T. Okitsu, T. Kawanishi, and S. Takeuchi, "Long-term in vivo glucose monitoring using fluorescent hydrogel fibers," Proceedings of the National Academy of Sciences of the United States of America, 2011, 108(33): 13399-13403.

[134] R. L. Fork, B. I. Greene, and C. V. Shank,
"Generation of optical pulses shorter than $0.1 \mathrm{psec}$ by colliding pulse mode locking," Applied Physics Letters, 1981, 38(9): 671-672.

[135] A. Penzkofer, "Passive Q-switching and modelocking for the generation of nanosecond to femtosecond pulses," Applied Physics B Photophysics and Laser Chemistry, 1988, 46(1): 43-60.

[136] L. Shi, T. Zhu, D. Huang, M. Liu, M. Deng, and W. Huang, "In-fiber whispering-gallery-mode resonator fabricated by femtosecond laser micromachining," Optics Letters, 2015, 40(16): 3770-3773.

[137] N. Zhang, K. Li, Y. Cui, Z. Wu, P. P. Shum, J. L. Auguste, et al., "Ultra-sensitive chemical and biological analysis via specialty fibers with built-in microstructured optofluidic channels," Lab on a Chip, 2018, 18(4): 655-661.

[138] P. Vaiano, B. Carotenuto, M. Pisco, A. Ricciardi, G. Quero, M. Consales, et al., "Lab on fiber technology for biological sensing applications," Laser \& Photonics Reviews, 2016, 10(6): 922-961. 